\title{
Cardiac tamponade with pneumothorax- An unusual life threatening complication following permanent pacemaker implantation.
}

\author{
RUPESH KUMAR ${ }^{1,2}$, Sanjib Rawat ${ }^{2}$, Vikram Halder², SHEENAM WALIA $^{2}$, \\ BANASHREE MANDAL ${ }^{2}$, and Shyam Thingnam ${ }^{2}$ \\ ${ }^{1}$ Postgraduate lnstitute of Medical Education and Research \\ ${ }^{2}$ Post Graduate Institute of Medical Education and Research
}

July 1, 2020

\begin{abstract}
Permanent pacemaker implantation is life saving but sometimes the procedure may itself become life threatening. Right ventricular perforation is a rare complication during pacemaker insertion that could be life-threatening and need immediate intervention. Chest X-ray, echocardiogram and computer tomogram of chest are used to diagnose lead migration and its complications. We present a rarest of the rare case in which a seventy six year old patient undergoing permanent pacemaker implantation suffered both iatrogenic cardiac injury leading to hemopericardium and pacemaker malfunction as well as injury to left pleura leading to massive pneumothorax and hemodynamic instability which was managed successfully.
\end{abstract}

Corresponding author with address - Dr Rupesh Kumar MCh, Associate Professor, department of cardiothoracic and vascular surgery, Room 4018, $4^{\text {th }}$ floor, Advanced Cardiac Center, Postgraduate institute of medical education and research, Chandigarh, Pin 160012, India. Email id- rkctvs@gmail.com Mobile- +919883690388

Source of funding - No grants, financial, technical or any other support was taken for this manuscript.

Article word count- 641 (excluding abstract and references)

Author contributions

Conceptualization: RK, VH, and SW. Data curation: RK and BM. Formal analysis: SR and SKST. Methodology: RK, BM, and VH. Project administration: RK and VH. Visualization: RK, BM, VH, SW, and BM. Writing original draft: RK and SKST. Writing - review and editing: RK, VH, BM, SR, SW,BM and SKST.

Ethics statement

All procedures performed in this study were in accordance with the ethical standards of the institutional and national research committee and with the 1964 Helsinki declaration and its later amendments or comparable ethical standards. For this study, formal consent was obtained.

Introduction

Permanent pacemaker implantation (PPI) is life saving but sometimes the procedure may itself become life threatening. Right ventricular perforation is a rare life threatening complication of PPI. Chest X-ray, echocardiogram and computer tomogram of chest are used to diagnose its complications. Cardiac chamber perforation is a rare complication of PPI that sometimes need immediate intervention in hemodynamically 
unstable patients. Most patients present with chest pain, dyspnea, profuse sweating and cold extremities. Chest X-ray, echocardiogram and computer tomogram of chest are the sequential tests to diagnose lead migration and its complications.

Case report

A seventy six year old man was referred to our emergency ward with complaints sudden hypotension, bradycardia and breathlessness following PPI for symptomatic bradyarrhythmia. On examination the patient was conscious, with profuse sweating. His pulse rate was 36/ minute, blood pressure of $75 / 44 \mathrm{~mm}$ of $\mathrm{Hg}$, cold extremities. The respiratory rate was 28 per minute, intercostals spaces in the left hemithorax appeared full of inspection and diminished breath sounds on auscultation. The PPI was not sensing even with the highest output set at the pulse generator. A chest x-ray was done which revealed pacemaker wire out of cardiac silhouette with left sided pneumothorax.(figure1) An intercostals chest tube of size 32Fr was inserted through which gush of air drained out. The patient was relieved of breathing difficulties to a great extent but the bradycardia and hypotension persisted. A transthoracic echocardiogram showed moderate pericardial effusion. The presence of pericardial effusion combined with loss of PPI to accelerate heart rate despite highest output led us to the suspicion of the pacemaker lead contact out of the cardiac chamber, hence a temporary pacemaker through femoral venous access was also inserted. An urgent CT scan was done which showed the lead end of the PPI out of the cardiac chamber and lying in the left pleural cavity piercing through it leading to iatrogenic pneumothorax. (Figure 2) As the lead was out of the cardiac chamber with moderate pericardial effusion a high suspicion of hemopericardium was made. The relatives were explained regarding the need of urgent surgical exploration for hemopericardium and need to remove PPI and replace it with epicardial pacemaker. Through median sternotomy, pericardiotomy was done and hemopericardium was drained out, it was further noted that the lead of the PPI was lying in the pericardium exiting through the diaphragmatic surface of the right ventricle and with its tip lying in the left pleural cavity piercing through it causing pneumothorax and hemodynamic compromise. (Figure 3) The pulse generator site on the right infraclavicular region was explored and the wire as gently pulled out. The rent over the right ventricle was sutured with no 4-0 polypropylene pledgeted sutures and hemostasis was secured. A new epicardial pacing wire was implanted over the anterior surface of the right ventricle and the other end taken out through the chest wall and connected with the pulse generator. It was checked and found to be working with minimum threshold and optimum voltage and output. The rest of the procedure was conducted in the usual fashion. The patient was extubated on the same day and was discharged on the third post operative day, the patient is on regular follow up for the last six months with no further cardiothoracic problems.

\section{Discussion}

Right ventricular perforation is a rare complication during PPI that sometimes could be life-threatening and need immediate intervention in hemodynamically unstable patients [1]. Postcardiac injury syndrome and stroke following PPI is also known [2]. Pneumothorax as a complication of vascular access during cardiac interventions have been reported [3]. The management of accidental and iatrogenic foreign body injuries to heart should be managed on priority as foreign bodies may manifest with infection, arrhythmia, or neurotic complications like cardiac tamponade [4]. Life threatening complications of like cardiac tamponade is a surgical emergency and urgent surgery is the only mode of therapy in these individuals[5].In a largescale, real-world experience of PPI in developed countries, the results indicate an increase in the incidence of in-hospital tamponade [6].Cardiac tamponade can occur secondary to perforation of the right ventricle during pacemaker electrode insertion and manipulation[7].Cardiac perforation usually occur more common through right ventricular apex as it is relatively thinner than the other implantable sites like septum or right ventricular outflow tract[8]. Low body mass index, age more than eighty years and prolonged use of steroids are the risk factors of cardiac chamber perforation [8]. Chest X-ray, echocardiogram and computer tomogram of chest are the sequential tests to diagnose lead migration and its complications [8].

Conclusion

PPI is a life saving procedure for several life threatening arrhythmias and symptomatic bradyarrhythmia 
but some tomes the procedure may itself lead to complication which may at times be life threatening. Hence the PPIs must be placed very cautiously, too much effort of rapid negotiation into the cardiac chamber may lead to the right ventricular perforation and even iatrogenic pneumothorax leading to severe hemodynamic compromise as seen in our case. Timely diagnosis and prompt management of this complication is life saving.

Acknowledgement

No grants, financial, technical or any other support was taken by any means for this manuscript.

Disclosures

No financial, property or intellectual aid was taken from any commercial organization for the manuscript publication.

\section{References:}

1. Khalid M, Murtaza G, Ayub MT, Ramu V, Paul T. Right Ventricle Perforation Post Pacemaker Insertion Complicated with Cardiac Tamponade. Muacevic A, Adler JR, eds. Cureus. 2018;10(3):e2266.

2. BialyCaitlin, WeeEdmund, Nizamuddin.Postcardiac injury syndrome and stroke following pErma nent pacemakerinsertion. BMJCaseRep. 2017;Aug11;2017. doi:10.1136/bcr-2017-220572

3. Ogunbayo, Gbolahan O. et al. Incidence, predictors, and outcomes associated with pneumothorax during cardiac electronic device implantation: A 16-year review in over 3.7 million patients Heart Rhythm , Volume 14, Issue 12, $1764-1770$

4. Kumar R, Rana SS, Kumar S, Das D, Datta M. Management of Accidental and Iatrogenic Foreign Body Injuries to Heart- Case Series. Journal of Clinical and Diagnostic Research : JCDR. 2017;11(3):PE01-PE04. doi:10.7860/JCDR/2017/23847.9336.

5. Das D, Datta M, Dey S, Parida J, Kumar R, Pande A. An Unusual Cause of Cardiac Tamponade during Cardiac Catheterization Study. Case Reports in Cardiology. 2014;2014:652592.

doi:10.1155/2014/652592.

6. Kasra Moazzami, Elena Dolmatova, Neil Kothari, Victor Mazza, MarcKlapholz, Alfonso

H. Waller. Trends in Cardiac Tamponade Among Recipients of Permanent Pacemakers in the United States: From 2008 to 2012 JACC: Clinical Electrophysiology Jan 2017, 3 (1) 41

46; DOI:10.1016/j.jacep.2016.05.009

7. Gershon T, Kuruppu J, Olshaker JJ. Delayed cardiac tamponade after pacemaker insertion. J. Emerg Med. 2000 Apr;18(3):355-9.

8. Vanezis AP, Prasad R, Andrews R. Pacemaker leads and cardiac perforation. JRSM Open. 2017;8(3):2054270416681432. doi:10.1177/2054270416681432.

Figure legends

Figure1

Chest $\mathrm{x}$ ray showing PPI out of cardiac silhouette and its tip in left hemithorax causing 
pneumothorax.

Figure 2

Computer tomography of chest showing collapsed left lung due to pheumothorax.

Figure 3

Intra-operative finding showing PPI lead perforated the right ventricular apex( shown with a pair of forceps).

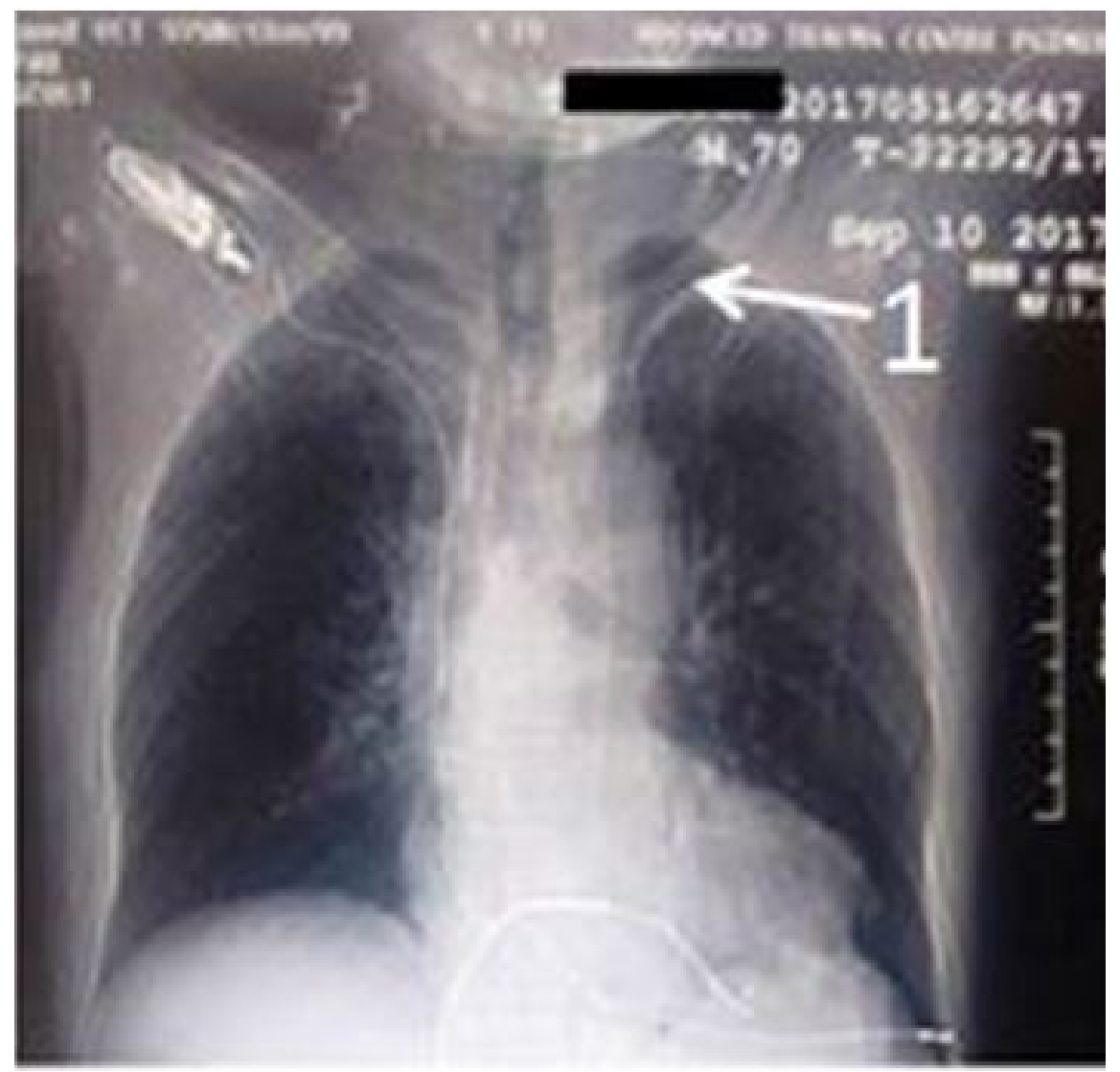




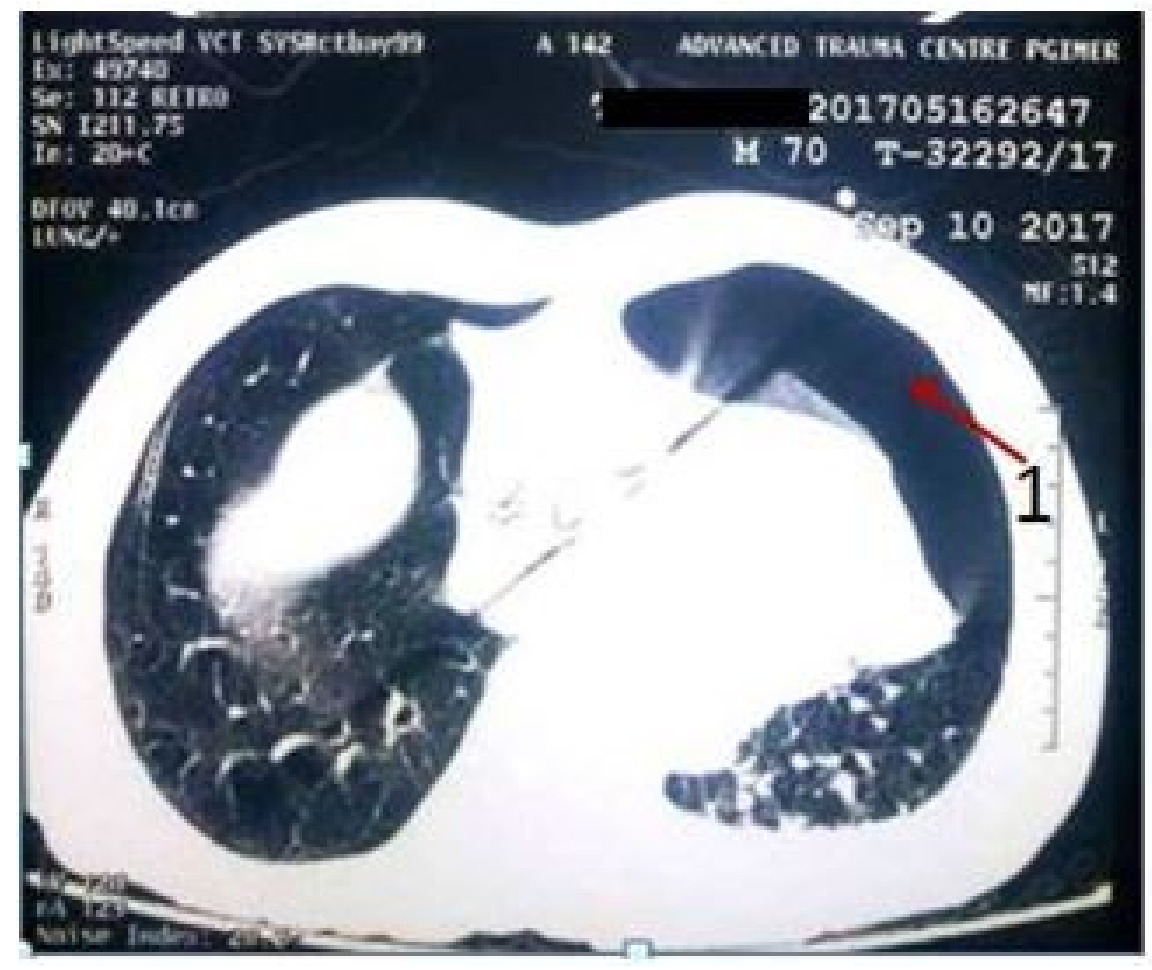




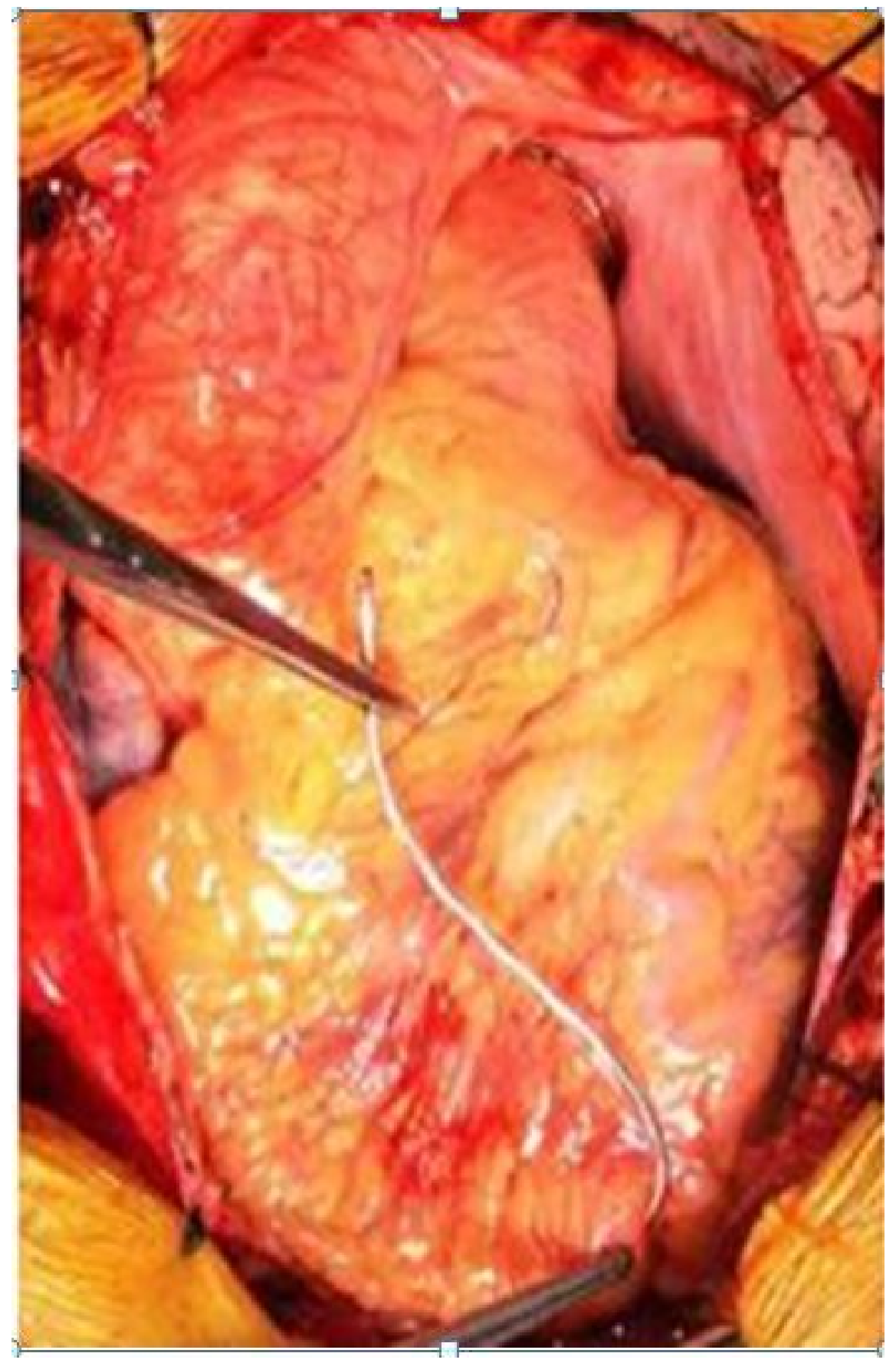

